\title{
Fragile X-associated tremor/ataxia syndrome
}

INSERM

\section{Source}

INSERM. (1999). Orphanet: an online rare disease and orphan drug data base. Fragile Xassociated tremorlataxia syndrome. ORPHA:93256

Fragile X-associated tremor/ataxia syndrome (FXTAS) is a rare neurodegenerative disorder characterized by adult-onset progressive intention tremor and gait ataxia. 